\title{
Discrimination of Dispersive Materials from Radar Signals USING $\mathbf{Q}^{*}$
}

\author{
Chun an Tsai ${ }^{1}$, Rebecca Ghent ${ }^{1,2}$, \\ AleXANDer Boivin ${ }^{2}$, and Dylan Hickson ${ }^{3}$ \\ ${ }^{1}$ Department of Physics, University of Toronto, Toronto, Canada - \\ ctsai@physics.utoronto.ca, ghentr@es.utoronto.ca \\ (C. Tsai is the Corresponding Author) \\ 2 Department of Earth Sciences, University of Toronto, Toronto, Canada - \\ alex.boivin@mail.utoronto.ca \\ ${ }^{3}$ Centre for Research in Earth and Space Science, York University, \\ Toronto, Canada - dylan.hickson@gmail.com
}

\begin{abstract}
Using a combination of laboratory measurements and modeling results, we demonstrate the potential of distinguishing two dispersive materials by estimating quality factor $\left(Q^{*}\right)$ using radar signals at two different frequencies. Here, we report on new complex dielectric permittivity measurements of a pulp sample mainly composed of pyrite (25\%) and quartz (55\%) from a massive sulphide mine, which shows frequency-dependent permittivity, and of a calcium-rich montmorillonite sample (STx-1b) for comparison. We made these measurements using the coaxial transmission line technique. To understand the dispersion observed in both samples, we fitted the measured complex permittivities using the Cole-Cole model to obtain the relaxation times that best represent the dielectric losses. We chose montmorillonite as the "control" material because it readily absorbs water, which has well-known dielectric relaxation mechanisms, thus providing a means of testing whether the pulp sample relaxations could be distinguished from those caused by adsorbed water. Our inverted montmorillonite relaxation times show one interlayer-water relaxation and one free water relaxation, as expected for this clay structure. By contrast, the pyrite-quartz sample shows intrinsic dispersion that is independent of the influence of water. The measurements show that the two materials have opposite concavity in the attenuation v.s. frequency plot, which can be detected using $Q^{*}$ in principle. Using these results, we conducted a series of 3D Finite-Difference-Time-Domain (FDTD) simulations in a cross-hole setup to
\end{abstract}


explore the effects of the observed dispersion on material detectability. We show that it is possible to distinguish intrinsically dispersive materials from those that are simply wet.

Keywords: Ground penetrating radar; Dispersion; Complex permittivity measurement; Spectral decomposition; Quality factor.

\section{Introduction}

Ground penetrating radar (GPR) is a nondestructive measurement technique which uses the transmission or reflection of electromagnetic (EM) waves to locate targets, anomalies or interfaces beneath or within natural or artificial surfaces. One basic assumption of GPR surveys is that subsurface features return reflections that are replicas of the transmitted signal with lower amplitudes. This implies that electrical properties of materials are independent of frequency within the frequency range of GPR, which is often referred to as the "GPR plateau" [1]. While this assumption holds true for most materials in the frequency band of GPR operation, some materials, especially materials that contain water, have frequency-dependent dielectric permittivities. As the complex permittivity varies with frequency, both the velocity and attenuation of the EM waves also change. This type of dispersion is categorized as physical property dispersion [2]. Scattering from heterogeneities in the subsurface can cause frequency-dependent attenuation as well. The attenuation measured in the field is mainly the combination of intrinsic dispersion and scattering dispersion, and it is difficult to isolate the two. There are several parameters used to characterize frequency-dependent attenuation. Turner and Siggins [3] show that, similar to seismic wave analysis [4], we can use a constant $\mathrm{Q}^{*}$ parameter to characterize materials with frequency-dependent attenuation in GPR surveys. Bradford [5] defines a more general dispersion parameter D that includes all frequency-dependent attenuation. One of the methods to extract $\mathrm{Q}^{*}$ from radar signals is the spectral shift method [6]. As the signal propagates, the peak frequency shifts lower from the original source value, and the difference can be used to estimate $Q^{*}$ of the material.

In previous studies, water content was reported to be the major cause of dispersion. Therefore materials containing variable amounts of water, 
such as clay [7-10] and concrete [11-13] are of major interest in the study of frequency-dependent attenuation of radar waves. Our goal for this project was to identify a dispersion behaviour that is independent of the influence of water and determine whether the difference can be identified from radar signals [14]. Since clay is a typical soil material that can absorb a large amount of water, we chose a montmorillonite sample as a reference material to characterize the influence of water on its dielectric properties. We measured the complex permittivity of the montmorillonite at varying moisture levels, then fitted the data with a multi-pole Cole-Cole model to obtain the dielectric relaxations responsible for the dispersion. We also fitted the measurements of a pulp sample that is mainly composed of pyrite and quartz from the LaRonde massive sulphide mine that shows a different dispersive behaviour from the montmorillonite sample. Then, we show with numerical simulations that it is possible to distinguish between these two types of dispersive behaviours by comparing the $\mathrm{Q}^{*}$ values at two frequencies. We believe that this technique expands the potential application of radar signals in material characterization.

\section{Theory}

\subsection{Electromagnetic wave propagation in dielectrics}

A GPR system generates EM waves that are either pulsed or continuous, in the frequency range of $10 \mathrm{MHz}$ to a few $\mathrm{GHz}$. The EM waves penetrate and propagate in the dielectric, then are transmitted or reflected to the receiving antenna, depending on the setup of the survey. The propagation of EM waves in dielectrics is controlled by many factors, such as antenna characteristics, scattering, attenuation, etc. Here we focus on the study of complex relative permittivity, which characterizes the speed and attenuation of EM waves during propagation. Complex relative permittivity consists of a real and an imaginary part:

$$
\epsilon^{*}(\omega)=\epsilon^{\prime}(\omega)-j \epsilon^{\prime \prime}(\omega)
$$

where $\epsilon^{*}, \epsilon^{\prime}$ and $\epsilon^{\prime \prime}$ are the complex, real, and imaginary components of relative permittivity, respectively. For most materials, the complex permittivity is dependent on frequency $f$, and $\omega=2 \pi f$. In this study, we 
assume that the relative magnetic permeability is 1 for all materials. This is quite accurate for most materials and a common assumption made in many studies. The electric field of a plane wave at position $\vec{r}$ and time $t$ is given by

$$
\vec{E}(\vec{r}, t)=\overrightarrow{E_{0}} e^{j(\vec{k} \cdot \vec{r}-\omega t)}
$$

where $\vec{E}_{0}$ is the constant amplitude vector and $\vec{k}$ is the complex wave vector. The complex wave vector is given by

$$
\vec{k}=(\beta-j \alpha) \hat{k}
$$

where the unit vector $\hat{k}$ denotes the direction of propagation of the wave. The phase constant $\beta$ and attenuation constant $\alpha$ are defined as

$$
\begin{aligned}
& \beta=\frac{\omega}{c_{0}} \sqrt{\frac{\epsilon^{\prime}}{2}\left[\sqrt{1+\left(\frac{\epsilon^{\prime \prime}}{\epsilon^{\prime}}\right)^{2}}+1\right]} \\
& \alpha=\frac{\omega}{c_{0}} \sqrt{\frac{\epsilon^{\prime}}{2}\left[\sqrt{1+\left(\frac{\epsilon^{\prime \prime}}{\epsilon^{\prime}}\right)^{2}}-1\right]}
\end{aligned}
$$

where $c_{0}$ is the speed of EM waves in free space $\left(\sim 3 \times 10^{8} \mathrm{~m} / \mathrm{s}\right)$. We can multiply $\alpha$ by 8.686 to convert to the more commonly used units of $\mathrm{dB} / \mathrm{m}$. The phase velocity of the wave can is given by $v=\omega / \beta$. Note that when the imaginary part of the relative permittivity is small compared to the real part, the phase velocity can be approximated as $v=c_{0} / \sqrt{\epsilon^{\prime}}$.

In general, attenuation and phase velocity of EM waves are frequency dependent in real materials. One way to characterize frequency-dependent attenuation is using quality factor $Q$, which is defined as

$$
Q=\frac{\omega}{2 v \alpha}
$$

This parameter describes the ratio of energy stored to energy dissipated in one cycle. For most materials encountered in GPR surveys, the frequency-dependent attenuation is linear in the relevant frequency bandwidth [3]. Thus we can approximate the linear region with the best fit line described by 


$$
\alpha=\alpha_{0}+\frac{\omega-\omega_{0}}{2 v Q^{*}}
$$

where $\alpha_{0}$ is the attenuation at a reference frequency $\omega_{0}$. As radar signals propagate in dispersive materials, the peak of the amplitude spectrum shifts due to frequency-dependent attenuation, and we can measure $Q *$ from the amount of frequency shift. The relation between $Q *$ and the corresponding spectral shift of a Ricker wavelet is given by $[5,6]$

$$
\frac{1}{Q^{*}}=\frac{4}{t} \frac{\left(\omega_{m}^{2}-\omega_{t}^{2}\right)}{\omega_{m}^{2} \omega_{t}}
$$

where $\omega_{m}$ is the spectral maximum of the source and $\omega_{t}$ is the spectral maximum at time $t$.

\subsection{Models of dielectric dispersion}

In this paper we study the frequency-dependent complex permittivity with the Debye model [15] and the Cole-Cole [16] model. The Debye model describes how pure polar molecules (dipoles) behave in the presence of an electric field. Instead of reacting instantaneously, the dipoles need a period of time, called the relaxation time, to reorient themselves along the direction of the applied electric field. To be more specific, relaxation time $\tau$ is defined as the time required to reach $1 / e$ ( $e$ being Euler's number) of the original polarization when the field is removed; and the frequency dependence of the permittivity is given as:

$$
\epsilon^{*}(\omega)=\epsilon_{\infty}+\sum_{n=1}^{N} \frac{\epsilon_{s, n}-\epsilon_{\infty}}{1+j \omega \tau_{n}}-j \frac{\sigma_{d c}}{\omega \epsilon_{0}}
$$

where $\epsilon_{\infty}$ is the relative permittivity at infinite-frequency limit, $\epsilon_{s}$ is the relative permittivity at static limit, $\tau$ is the relaxation time, and $\sigma_{d c}$ is the Direct Current (DC) electrical conductivity. The summation symbol indicates that there may be more than one type of polar molecule involved. It is often useful to write out the real and the imaginary parts of (9) explicitly:

$$
\epsilon^{*}=\epsilon^{\prime}-j \epsilon^{\prime \prime}
$$




$$
\epsilon^{\prime}=\epsilon_{\infty}+\frac{\epsilon_{s}-\epsilon_{\infty}}{1+\omega^{2} \tau^{2}} \quad \epsilon^{\prime \prime}=-\frac{\omega \tau\left(\epsilon_{s}-\epsilon_{\infty}\right)}{1+\omega^{2} \tau^{2}}+\frac{\sigma_{d c}}{\omega \epsilon_{0}}
$$

The Debye model has also been modified into various forms to describe experimental results. The most general form is the Havriliak-Negami (HN) relaxation, which has two additional parameters compared to Debye's model. It was first used to describe dielectric relaxation in polymers [17]. The one-pole $\mathrm{HN}$ relaxation is:

$$
\epsilon^{*}=\epsilon_{\infty}+\frac{\epsilon_{s}-\epsilon_{\infty}}{\left[1+(j \omega \tau)^{\gamma}\right]^{\nu}} \quad 0 \leq \gamma, \nu \leq 1
$$

If $\beta=1$, $\mathrm{HN}$ relaxation reduces to a Cole-Cole relaxation. If $\gamma=1$, $\mathrm{HN}$ relation reduces to a Cole-Davidson relaxation. The Debye relaxation is a special case of HN relaxation where $\gamma=\nu=1$. The parameter $\gamma$ controls the "broadness" of the relaxation peak. The smaller $\gamma$ is, the broader the relaxation peak would be on the frequency axis. On the other hand, $\nu$ describes the "asymmetry" of the relaxation peak. When $\nu=1$, the relaxation peak is symmetrical.

\section{Methodology}

In order to quantitatively describe the dispersion caused by water, we measured the complex permittivity of a clay sample under various moisture levels. We chose the calcium-rich montmorillonite as our reference sample because its structure is well-understood. We also measured the complex permittivity of pulp prepared from drilling core samples that is mostly composed of quartz and pyrite. We then fitted the permittivity data to the Cole-Cole model for interpretation of the relaxation mechanisms.

\subsection{Laboratory measurements}

We measured the permittivity of two powdered samples: calcium-rich montmorillonite (STx-1b) from the Clay Minerals Society and a pulp sample (pyrite and quartz) provided by Agnico Eagle Mines Ltd. There is about $25 \%$ pyrite $\left(\mathrm{FeS}_{2}\right)$ and $55 \%$ quartz in the sample from X-ray 
fluorescence results. The samples were measured using the coaxial transmission line technique. We used the Keysight E5071C ENA series vector network analyzer (VNA) that measures from $300 \mathrm{kHz}$ to $14 \mathrm{GHz}$ in combination with METAS VNA Tools II software. Due to the length of the sample holder and the large uncertainty in the low-frequency range, we only used measurements from $50 \mathrm{MHz}$ to 8.5 GHz. The software automatically calculates the uncertainty in measurements [18]. Two Maury Microwave SC-35 cables were connected to the two ports of the VNA, and each cable was connected to a 3.5 $\mathrm{mm} / 14 \mathrm{~mm}$ adapter. The $14 \mathrm{~mm}$ side was then connected to the sample holder, which has a length of $15 \mathrm{~cm}$. The GR-900LZ sample holder is a cylindrical tube that has two parts: the outer conductor and the inner conductor. The inner diameter of the outer conductor is $14 \mathrm{~mm}$, and the outer diameter of the inner conductor is $6 \mathrm{~mm}$. Prior to each measurement, the VNA and the corresponding setup were calibrated using the full two-port Through-Reflection-Match (TRM) method [19]. The input and output signals from both ports are measured to determine the real and imaginary parts of the S-parameters. The complex permittivity can be calculated from the S-parameters using the non-iterative stable transmission/reflection method for low-loss material [20-22] The magnetic permeability $\mu_{r}$ is assumed to be 1 .

To observe the effect of water on permittivity more clearly, we first baked the clay sample at $115{ }^{\circ} \mathrm{C}$ for 48 hours [23] in order to remove the surface (free) water and to keep the interlayer water $[24,25]$. We also prepared a "moisture chamber" to achieve various moisture content. The moisture chamber is a sealable box with water covering the bottom. The samples were placed in a aluminum pan that was raised above the water surface. The sealable box ensures that water vapour can diffuse into the sample evenly. We varied the time the samples spend in the box to control the moisture content. We measured the baked sample first, then placed it into the moisture chamber for 3, 5, 7, 15, and 24 hours. We baked the samples for at least 24 hours between each measurement to ensure that the water content is entirely controlled by the time the samples spend in the moisture chamber. We calculated the water content by weight percentage. Instead of performing the same series of moisture measurements, we measured the pyrite-quartz sample under ambient and baked conditions only. 
Since dielectric permittivity varies as a function of density, the data were normalized to a bulk density of $1.60 \mathrm{~g} / \mathrm{cm}^{3}$ as shown in (13) [26-28]:

$$
\epsilon_{\text {norm }}^{*}=\epsilon^{*}(1.92)^{d_{n}-d}
$$

where $\epsilon^{*}$ is the complex permittivity, $d_{n}=1.60 \mathrm{~g} / \mathrm{cm}^{3}$, and $d$ is the bulk density of the measured sample.

\subsection{Decomposition of relaxation poles}

We inverted the complex permittivity measurements to fit the Cole-Cole model with a DC conductivity term:

$$
\epsilon^{*}=\epsilon_{\infty}+\frac{\epsilon_{s 1}-\epsilon_{\infty}}{1+\left(j \omega \tau_{1}\right)^{\alpha_{1}}}+\frac{\epsilon_{s 2}-\epsilon_{\infty}}{1+\left(j \omega \tau_{2}\right)^{\alpha_{2}}}-j \frac{\sigma_{d c}}{\omega \epsilon_{0}} .
$$

The best fit to the baked clay sample was achieved using a 1-pole model while the moist sample was best fit using a 2-pole model, suggesting one and two relaxation mechanisms, respectively. The number of relaxation poles we found to best fit the measured data is also consistent with the structure of montmorillonite. The interlayer bound water should be retained at our drying temperature, which was shown in previous studies to possess a different relaxation time from free water $[10,29]$. To invert for the parameters, we used a Levenberg-Marquardt scheme to perform least-squares fitting using the Lmfit module in Python [30].

\subsection{Numerical simulation}

In order to investigate the possibility of using dispersive properties to enhance GPR surveys, we performed numerical simulations based on the inversion of results calculated using the software gprMax [31]. GprMax is an open source software that simulates electromagnetic wave propagation by solving Maxwell's equations in a three-dimensional (3D) spatial domain using a Yee-grid scheme [32] and the finite-difference time-domain (FDTD) method. To handle dispersive materials, the software provides built-in Debye, Drude, and Lorentz models, for which arbitrary numbers of relaxation poles can be added to the simulation. The Cole-Cole model cannot be directly implemented into FDTD code. 
Therefore, we use multi-Debye relaxations to approximate the inversion using the Cole-Cole model [33]. The number of Debye poles was chosen so that the Debye inversion produces the closest residual with our original Cole-Cole inversion.

The 3D simulation space we used is $1.4 \mathrm{~m} \times 0.4 \mathrm{~m} \times 0.4 \mathrm{~m}$, with a spatial discretization of $4 \mathrm{~mm}$ in all three coordinates. The time-step $(7.7$ ps) used was derived from the 3D Courant-Friedrichs-Lewy condition:

$$
\Delta t \leq \frac{1}{c \sqrt{\frac{1}{(\Delta x)^{2}}+\frac{1}{(\Delta y)^{2}}+\frac{1}{(\Delta z)^{2}}}}
$$

where $\Delta x, \Delta y$ and $\Delta z$ represent the spatial discretization in the $x, y$, and $z$ directions, respectively. We used a simplified cross-hole setup with two Hertzian dipoles as the antennas. A schematic section of the simulation domain is shown in Figure 1. The transmitting antenna is located at (x, $\mathrm{y}, \mathrm{z})=(0.1 \mathrm{~m}, 0.2 \mathrm{~m}, 0.2 \mathrm{~m})$ and the receiving antenna is located at $(\mathrm{x}, \mathrm{y}$, $\mathrm{z})=(0.8 \mathrm{~m}, 0.2 \mathrm{~m}, 0.2 \mathrm{~m})$. The source waveform is a Ricker wavelet with a center frequency of $400 \mathrm{MHz}$ and $1200 \mathrm{MHz}$, polarized in the z-direction. A 10-cell perfectly-matched-layer (PML) is implemented on all faces of the simulation domain to prevent reflections back into the dielectric.

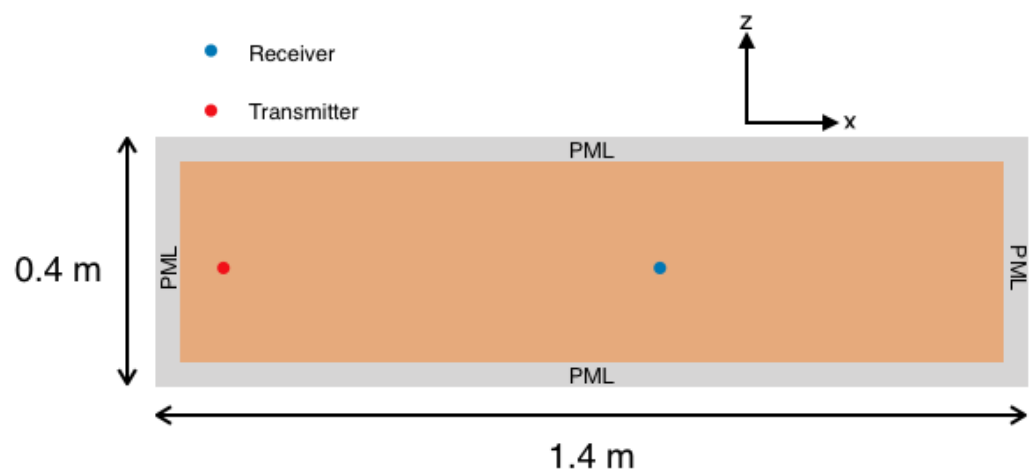

Figure 1: An $\mathrm{X}-\mathrm{Z}$ plane illustration of the simulation setup, in $y=0.2$. The transmitting antenna is located at $(\mathrm{x}, \mathrm{y}, \mathrm{z})=(0.1 \mathrm{~m}, 0.2 \mathrm{~m}, 0.2 \mathrm{~m})$ and the receiving antenna is located at $(\mathrm{x}, \mathrm{y}, \mathrm{z})=(0.8 \mathrm{~m}, 0.2 \mathrm{~m}, 0.2 \mathrm{~m})$. The grey area represents the PML that prevent reflections back into the simulation space. 


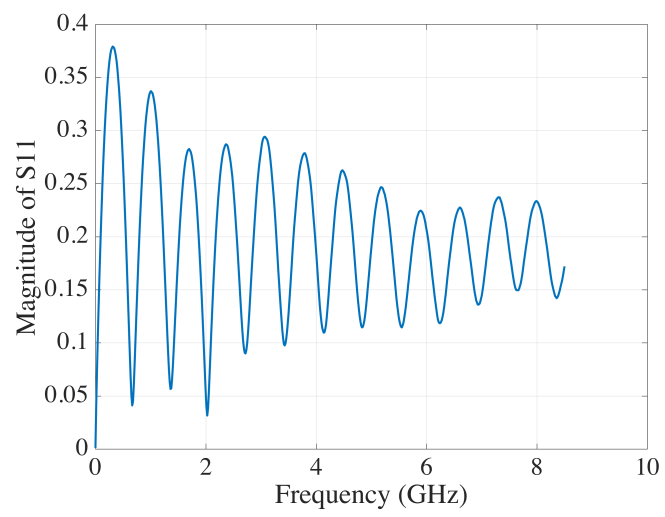

(a)

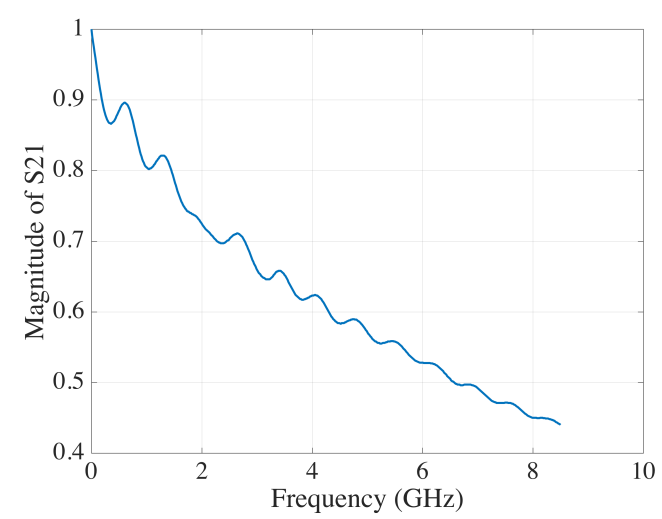

(c)

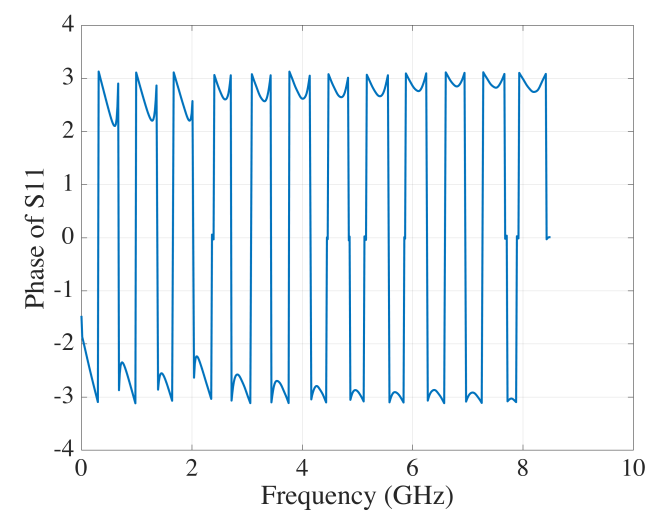

(b)

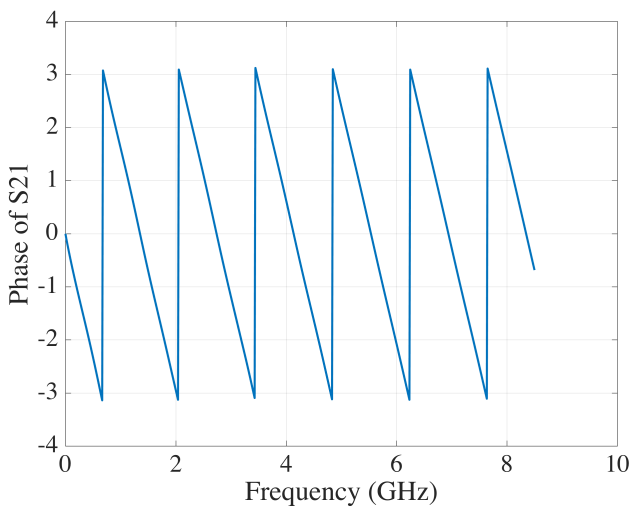

(d)

Figure 2: The magnitude and phase measurements of $S_{11}$ and $S_{21}$ from the baked montmorillonite sample. (a) $S_{11}$ magnitude spectrum. (b) $S_{11}$ phase spectrum. (c) $S_{21}$ magnitude spectrum. (d) $S_{21}$ phase spectrum.

\section{Results and discussion}

\subsection{Measurements and inversions}

The complex permittivities were computed from the measured S-parameters using the aforementioned method. An example of the S-parameter measurement is shown in Figure 2. We used a 1-pole Cole-Cole model to fit the baked sample and a 2-pole Cole-Cole model to fit the samples containing moisture. The results are shown in Table 1. Knowing the possible relaxation mechanisms in a material is helpful 
for determining the number of poles to use for inversion. In our clay experiment, free and bound water are the two most likely causes of relaxation $[8,10]$, consistent with our two-pole fit.

Table 1: Inversion results of montmorillonite sample STx-1b under various moisture levels. The moisture content by wt\% corresponds to 3-hour, 5-hour, 7-hour, 15-hour and 24-hour period in the moisture chamber.

\begin{tabular}{l|cccccc}
\hline Moisture by wt\% & Baked & 3.06 & 4.48 & 5.88 & 7.29 & 9.89 \\
\hline$\epsilon_{\infty}$ & 3.20 & 3.30 & 3.48 & 3.47 & 3.64 & 3.80 \\
$\epsilon_{s 1}-\epsilon_{\infty}$ & 1.88 & 7.15 & 7.29 & 9.36 & 10.6 & 12.5 \\
$\tau_{1}(\mathrm{~ns})$ & 1.79 & 14.0 & 1.78 & 1.48 & 1.63 & 1.57 \\
$\alpha_{1}$ & 0.389 & 0.488 & 0.647 & 0.660 & 0.681 & 0.682 \\
$\epsilon_{s 2}-\epsilon_{\infty}$ & & 0.264 & 0.412 & 0.643 & 0.825 & 1.13 \\
$\tau_{2}(\mathrm{ps})$ & & 10.3 & 10.0 & 6.96 & 10.0 & 8.88 \\
$\alpha_{2}$ & & 0.850 & 1.00 & 1.00 & 1.00 & 1.00 \\
$\sigma_{d c}(\mathrm{mS} / \mathrm{m})$ & 0.618 & 0.220 & 1.38 & 2.11 & 2.68 & 4.57 \\
bulk density $\left(\mathrm{g} / \mathrm{cm}^{3}\right)$ & 0.816 & 0.833 & 0.833 & 0.853 & 0.871 & 0.874 \\
\hline rms error & 0.0122 & 0.00160 & 0.00632 & 0.00909 & 0.00974 & 0.0135 \\
\hline
\end{tabular}

We show the change in the residual from a 1 to a 3-pole model in Figure 3 , as an additional evaluation of the number of poles required for inversion; the figure shows that the addition of relaxation poles to the baked sample inversion does not result in a better fit. The extra poles have extremely small amplitudes compared to the amplitude of the original 1-pole model. The residuals for the inversions of the moisturized samples decrease from 1- to 3-Cole-Cole fits, but the decrease is less significant from 2 to 3 poles than from 1 to 2 poles. This level-off in the residual curve is not as evident in the 7 -hour and 15-hour experiment as in the 3- and 5-hour experiments. In the 24-hour case, however, we see the level-off in the misfit curve becomes more apparent again. We judge that our data are best fit by a Cole-Cole models with 2 poles, rather than 3. 


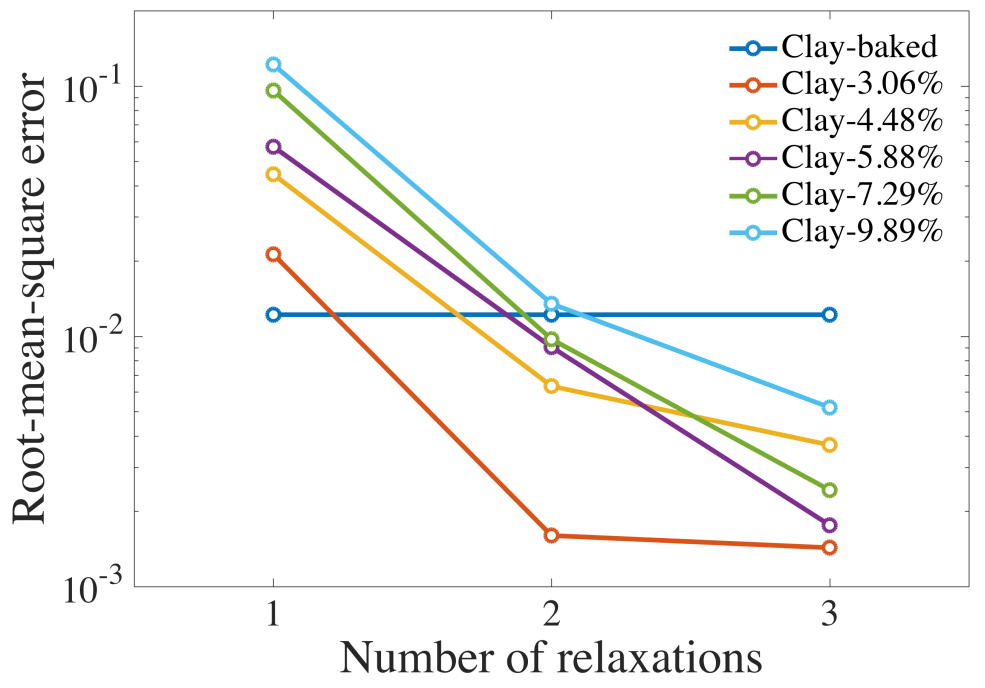

Figure 3: Root-mean-square error from inversion with 1-, 2-, and 3-pole Cole-Cole models.

Figure 4 shows the representative frequency spectra of the clay sample as well as the inverted curve (dark blue line) obtained from (14). The effect of each relaxation pole, and the DC conductivity, are also depicted as curves below the measured data. We can see that the DC conductivity only contributes to the lowest-frequency part of the spectra. The yellow curve contributes to the highest-frequency part of the spectra in the samples containing moisture. The corresponding relaxation time is around $9 \mathrm{ps}$, which agrees with the accepted value of the first relaxation pole of free water $[10,34]$. The blue curve aligns with the data in most of the frequency range, which corresponds to a relaxation time around $1.65 \mathrm{~ns}$. We attribute this to the relaxation caused by bound water in the interlayer between two 2:1 sheets. Each 2:1 sheet is composed of two tetrahedral sheets of silica sandwiching one central octahedral sheet of alumina [35]. Our value for this intermediate relaxation is lower than the previously reported values $[10,29]$, but is within the same order of magnitude. The inverted bound water relaxation time in the 3-hour case is much higher than the rest of the inversions. We believe that this is a numerical result rather than a physical one. 


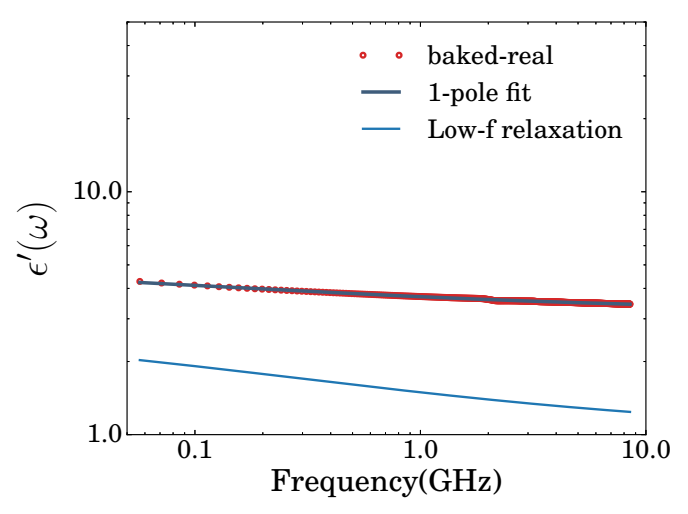

(a)

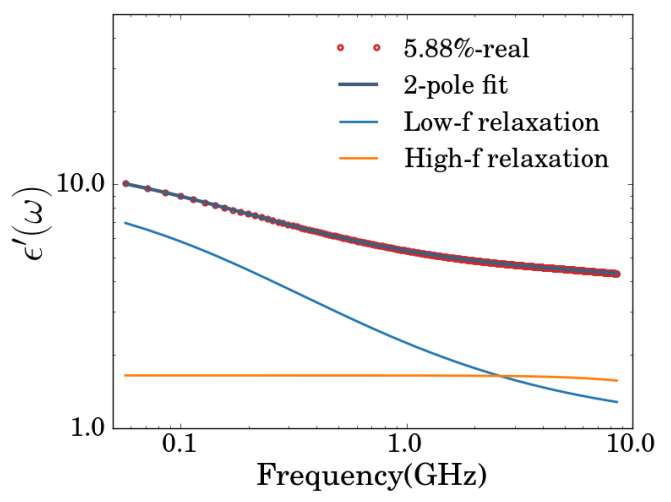

(c)

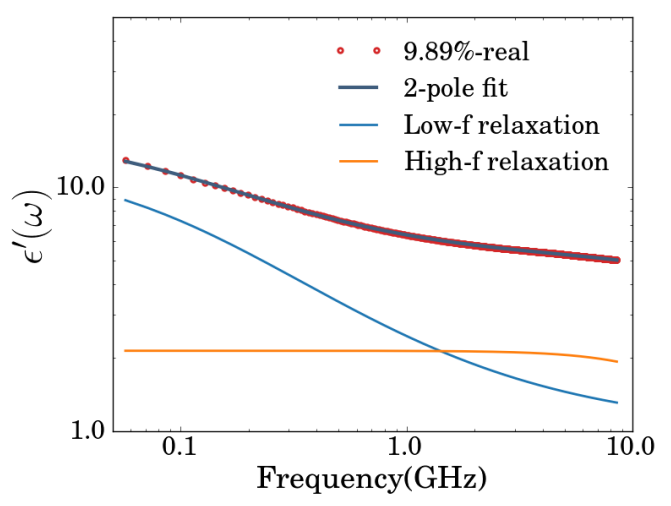

(e)

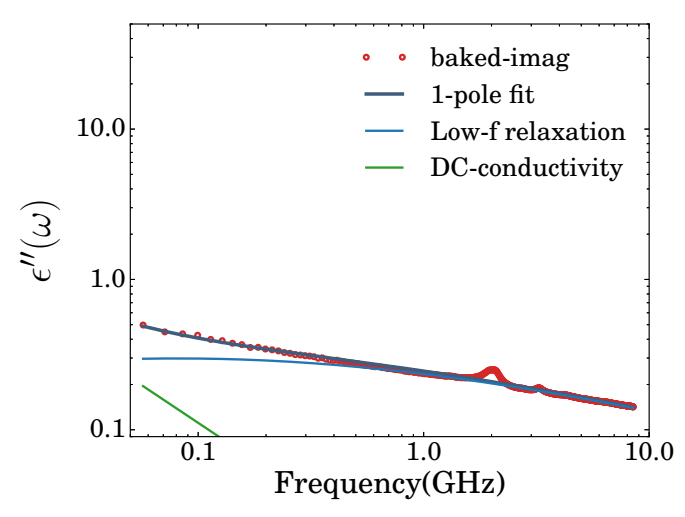

(b)

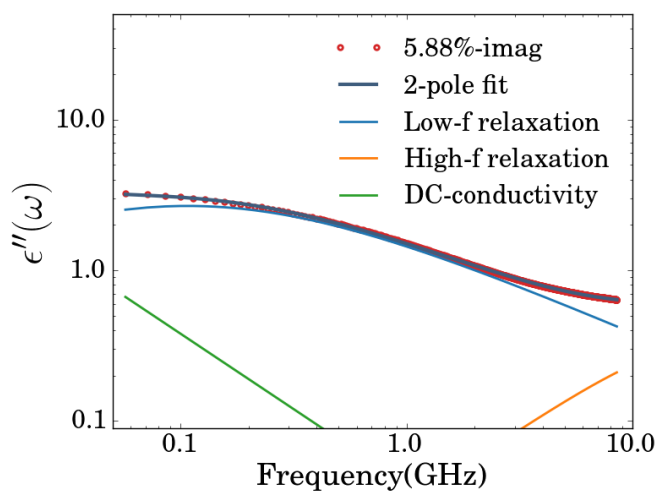

(d)

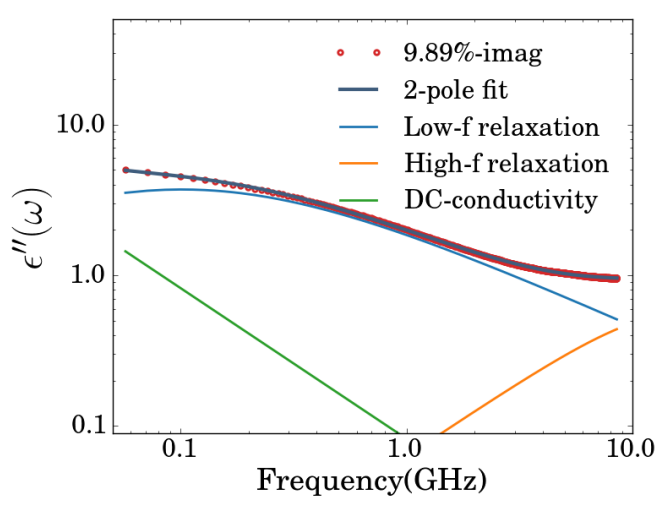

(f)

Figure 4: Complex permittivity and Cole-Cole model inversions of the montmorillonite sample under different moisture levels. The red dotted lines are the measurements and the dark blue lines are the inversion results using either 1-pole or 2-pole model. The orange and light blue lines represent high-frequency relaxation and low frequency relaxation, respectively. The green line represents the contribution from the DC conductivity. (a)-(b) baked clay. (c)-(d) 7-hr clay. (e)-(f) 24-hr clay. The measurement uncertainty above $800 \mathrm{MHz}$ is less than $5 \%$. 


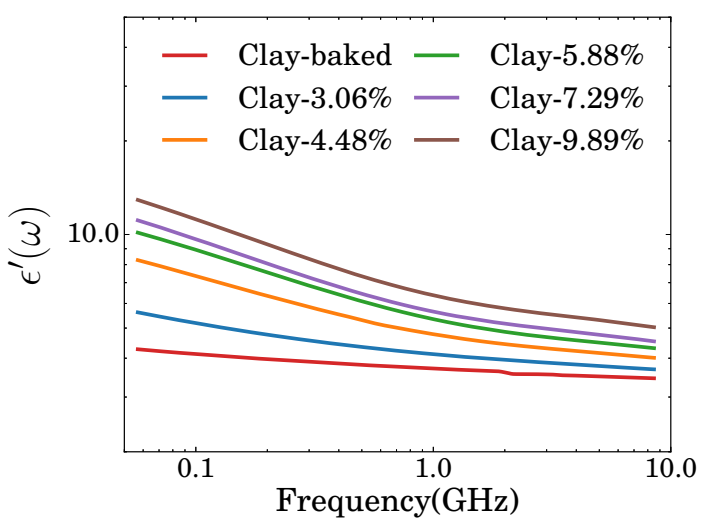

(a)

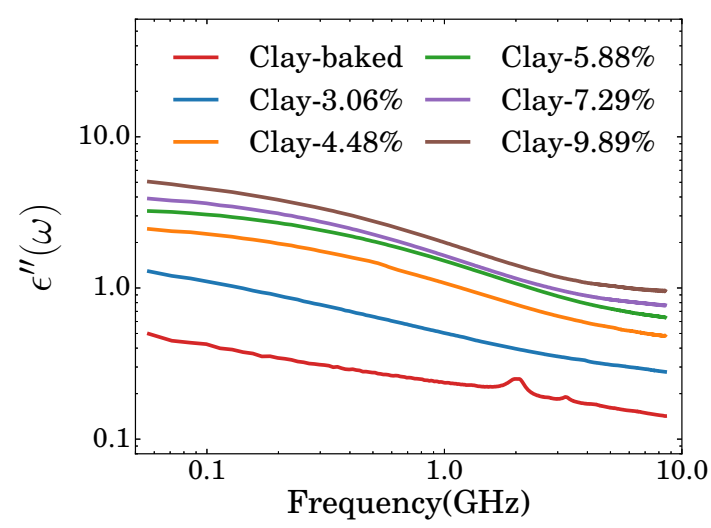

(b)

Figure 5: Comparison between the dielectric permittivity of the montmorillonite samples at various moisture levels shows a steady trend for both the real and the imaginary parts. (a) Real part of the permittivity measurement. (b) Imaginary part of the permittivity measurement.

Figure 5 shows the real and imaginary part of all the montmorillonite measurements. The trend from low to high moisture content is clear in both figures. As the moisture content increases, both the real and imaginary part increase gradually. The 3-hour curves do not show any sudden change from the rest of the measurements. Moreover, the shape of the imaginary part indicates that the 3-hour sample is in the "transition" from being better fitted with 1 pole to being better fitted with 2 poles. The inversion algorithm cannot fully capture this transition and produces a relaxation time that is not consistent with that of wetter samples as a result.

We measured/inverted the pyrite-quartz sample using the same procedure. However, the pyrite-quartz sample was baked at $250^{\circ} \mathrm{C}$ to ensure that all water was gone. The results for the baked sample are shown in Figure 6, where (a) is real part $\epsilon^{\prime}(\omega)$, and (b) is the imaginary part $\epsilon^{\prime \prime}(\omega)$. The pyrite-quartz sample clearly shows a dispersive behaviour that is distinct from that caused by water. The imaginary part of the pyrite-quartz sample goes up steadily from about $0.2 \mathrm{GHz}$ to the end of the spectrum, while the clays have a decreasing trend in the same frequency range. 


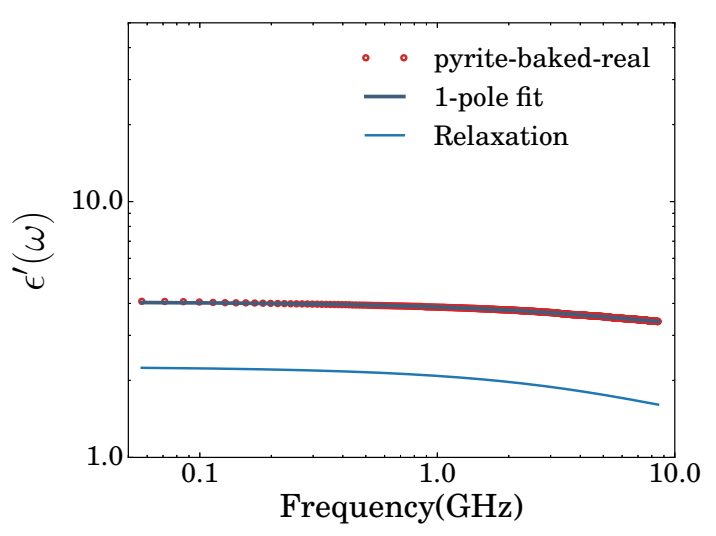

(a)

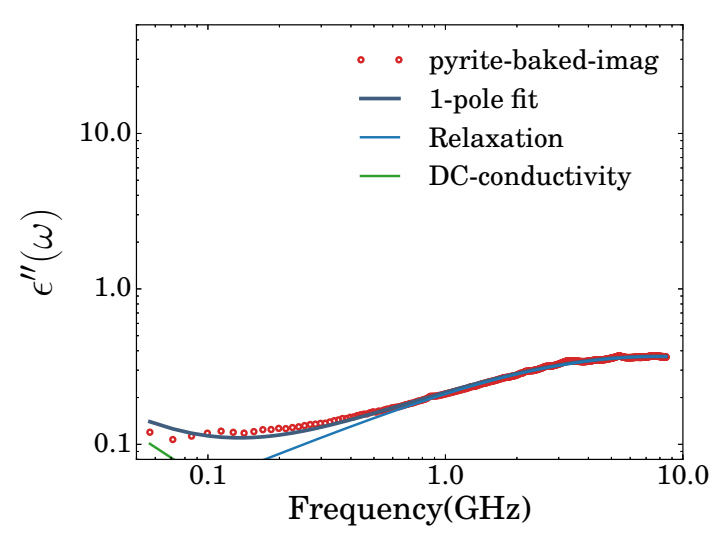

(b)

Figure 6: Complex permittivity and Cole-Cole model inversion of the baked pyrite-quartz sample. The red dotted lines are the measurements and the dark blue lines are the inversion results using 1-pole model. The light blue line represents relaxation peak determined from inversion. The green line represents the contribution from the DC conductivity. The measurement uncertainty above $800 \mathrm{MHz}$ is less than $5 \%$.

We plot the attenuation constant using the inverted parameters of the montmorillonite samples in Figure 7 . The increasing moisture content not only increases the magnitude of attenuation in montmorillonite, but also the slope with respect to frequency. Therefore, $\mathrm{Q}^{*}$ also decreases with increasing moisture content according to (7). Notice the slope is greatest in the low-frequency region. This suggests that we should obtain a larger $\mathrm{Q}^{*}$ using a low-frequency source, and a smaller $\mathrm{Q}^{*}$ with a high-frequency source. By contrast, the attenuation constant of pyrite-quartz sample is shown in Figure 8. Because of the opposite concavity of the attenuation curve, the pyrite-quartz should give a higher $\mathrm{Q}^{*}$ when using a low-frequency source compared to a high-frequency source. We can therefore distinguish between the two dispersive behaviours, one caused by water and one intrinsic to the pyrite-quartz sample, by estimating $\mathrm{Q}^{*}$ at two frequencies. This method essentially takes the second derivative of attenuation with respect to frequency into consideration, in contrast to the traditional constant- $\mathrm{Q}^{*}$ approach. 


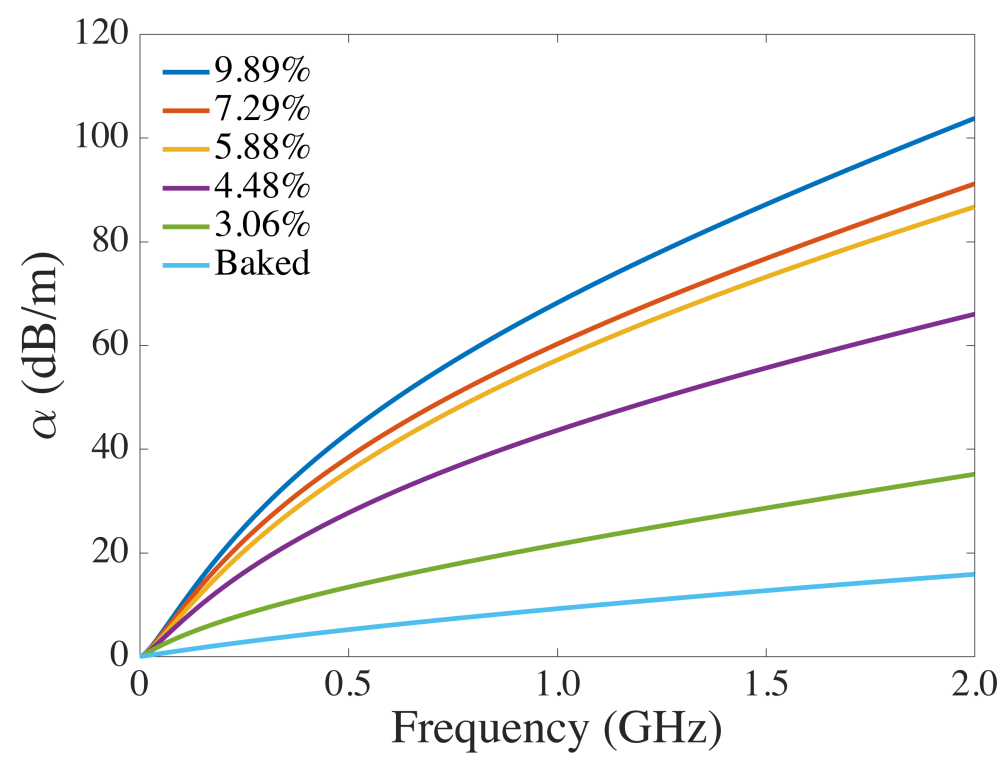

Figure 7: Attenuation $\alpha$ of the clay at varying moisture levels calculated from the Cole-Cole inversions.

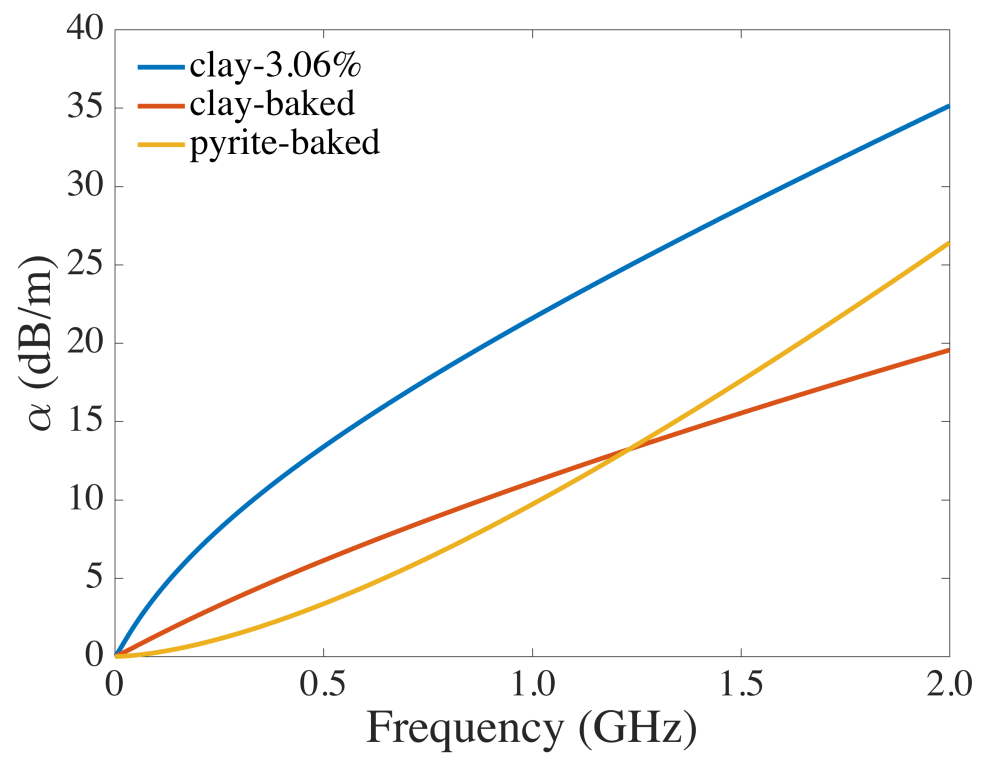

Figure 8: Attenuation $\alpha$ of clay at 3.06\%, baked clay, baked pyrite-quartz sample. 


\subsection{Simulation}

We performed numerical simulations using the permittivity data from the samples. In each simulation, the space is filled with one single material of interest (filled-space). We use a $1200 \mathrm{MHz}$ Ricker wavelet in one set of simulations and a $400 \mathrm{MHz}$ Ricker wavelet in another. The results from the simulations are shown in Figure 9 and Figure 10. While we inverted the permittivity data with Cole-Cole model to give physical interpretation of the parameters, we inverted the same data with Debye model in order to implement the simulations in gprMax. The number of Debye relaxation poles was chosen to best approximate the residuals from the Cole-Cole model inversion. In Figure 9 (a) and Figure 10 (a), the amplitudes of the received signals are scaled to better illustrate the difference in shape. We use the time difference between the positive peak of the received signal and the positive peak of the input signal to calculate the travel time. The spectral shifts of the signals are shown in Figure 9 (b) and Figure 10 (b). The peak frequency shifts more in samples with higher water content due to increase attenuation, as expected. With the travel time and spectral shift information, we can calculate $\mathrm{Q}^{*}$ for the materials at $1200 \mathrm{MHz}$ and $400 \mathrm{MHz}$ using (8).

We calculated $\mathrm{Q}^{*}$ using both the spectral shift method and the attenuation data from inverted parameters. The results are given in Table 2 and Table 3 . All the clay samples have a lower $\mathrm{Q}^{*}$ value obtained from attenuation at $400 \mathrm{MHz}$ compared to $1200 \mathrm{MHz}$. In contrast, the baked pyrite-quartz sample has a higher $\mathrm{Q}^{*}$ at $400 \mathrm{MHz}$ compared to $1200 \mathrm{MHz}$. This difference essentially gives information about the second derivative of attenuation with respect to frequency, which is the key to distinguish two different dispersive behaviours. We can observe a similar pattern in the calculation using the spectral shift method. This suggests the possibility of applying the concept in field data. However, there are several differences between the spectral shift calculation and the direct calculation from attenuation. Firstly, the spectral shift method overestimates $\mathrm{Q}^{*}$ for low-attenuation materials (baked clay and baked pyrite-quartz), and underestimates $\mathrm{Q}^{*}$ for the rest of the samples. Secondly, the $\mathrm{Q}^{*}$ values obtained from these two methods differ more at $1200 \mathrm{MHz}$ than at $400 \mathrm{MHz}$, especially for the high-attenuation materials. 


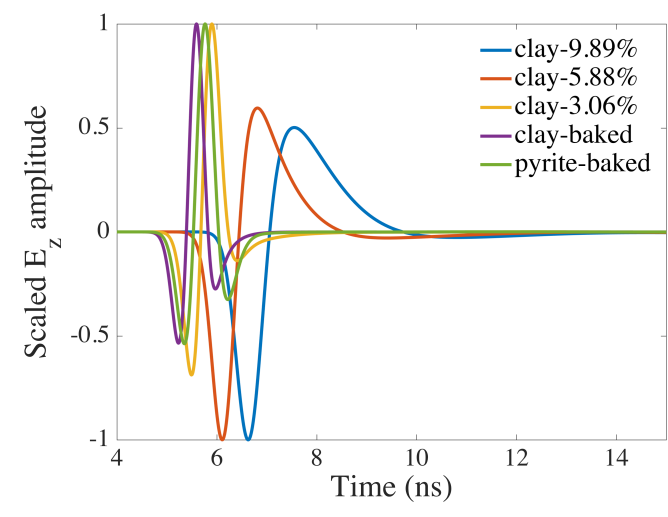

(a)

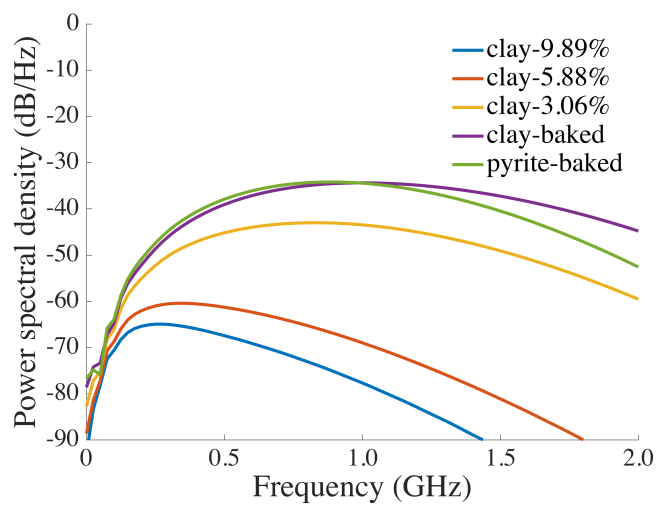

(b)

Figure 9: (a) Received signals in time domain and (b) corresponding power spectral density from a Ricker waveform centred at $1200 \mathrm{MHz}$. The materials are clays at $9.89 \%, 5.88 \%, 3.06 \%$ water content, baked clay, and baked pyrite.

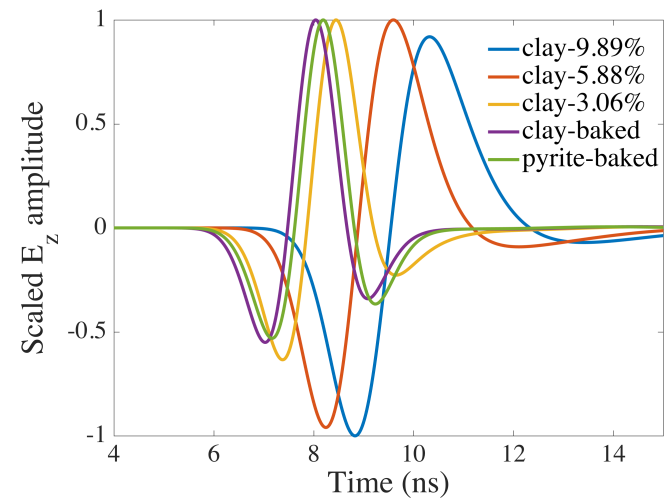

(a)

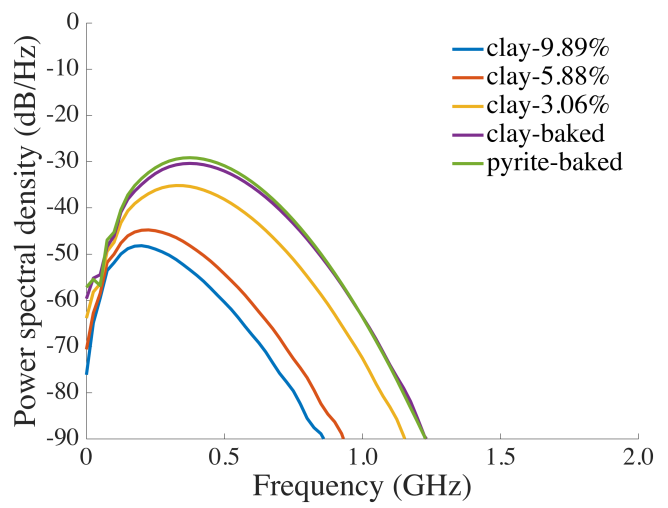

(b)

Figure 10: (a) Received signals in time domain and (b) corresponding power spectral density from a Ricker waveform centred at $400 \mathrm{MHz}$. The materials are clays at $9.89 \%, 5.88 \%, 3.06 \%$ water content, baked clay, and baked pyrite-quartz.

There are two factors controlling the spectral shift calculation: the travel time and the shift in spectral maximum. We compare the phase velocity calculated from travel time and the theoretical phase velocity for the $1200 \mathrm{MHz}$ case in Figure 11. The travel time estimated from peak-to-peak has to be larger than the actual value to produce a larger $\mathrm{Q}^{*}$. However, Figure 11 shows that the estimated phase velocities should 
Table 2: Comparison of $\mathrm{Q}^{*}$ calculated directly from attenuation curves and $\mathrm{Q}^{*}$ calculated from spectral shifts for the clay samples with different water contents.

\begin{tabular}{l|cccccc}
\hline Moisture by wt\% & Baked & 3.06 & 4.48 & 5.88 & 7.29 & 9.89 \\
\hline $1200 \mathrm{MHz}$ from $\alpha$ & 19.6 & 12.8 & 8.1 & 6.3 & 6.4 & 6.0 \\
$400 \mathrm{MHz}$ from $\alpha$ & 15.8 & 9.3 & 4.9 & 3.9 & 3.9 & 3.7 \\
$1200 \mathrm{MHz}$ from spectral shift & 22.7 & 11.7 & 5.1 & 3.2 & 3.2 & 2.9 \\
$400 \mathrm{MHz}$ from spectral shift & 19.8 & 8.9 & 3.8 & 3.1 & 2.6 & 2.8 \\
\hline
\end{tabular}

Table 3: Comparison of $\mathrm{Q}^{*}$ calculated directly from attenuation curves and $\mathrm{Q}^{*}$ calculated from spectral shifts for the pyrite-quartz sample.

\begin{tabular}{l|c}
\hline Pyrite-quartz & Baked \\
\hline $1200 \mathrm{MHz}$ from $\alpha$ & 11.5 \\
$400 \mathrm{MHz}$ from $\alpha$ & 19.3 \\
$1200 \mathrm{MHz}$ from spectral shift & 14.0 \\
$400 \mathrm{MHz}$ from spectral shift & 22.1 \\
\hline
\end{tabular}

give larger $\mathrm{Q}^{*}$ than that shown in Table 2 . The estimated phase velocities are lower than the actual values except for the baked clay sample. This means we already use longer travel times in the spectral shift method for high-attenuation materials. The problem therefore is caused by frequency estimation.

Although spectral maximum is used to calculate $Q^{*}$ in [5], centroid frequency is used in [6] instead. The amplitude spectrum of the clay sample with $9.89 \%$ is shown in Figure 12 . Clearly the spectral maximum is lower than the centroid frequency of the spectrum. As a result, the frequency shift appears to be larger, and causes the spectral shift method to produce a lower $\mathrm{Q}^{*}$. In addition, the spectral maximum of the 400 $\mathrm{MHz}$ spectrum is closer to its centroid frequency than the $1200 \mathrm{MHz}$ spectrum. Therefore the $400 \mathrm{MHz}$ estimation of $\mathrm{Q}^{*}$ is closer to the $\mathrm{Q}^{*}$ obtained directly from $\alpha$. Calculating the centroid frequency requires knowing the source spectrum; however, measuring source spectrum can be difficult in the field due to noise. Using the spectral maximum avoids this problem. 


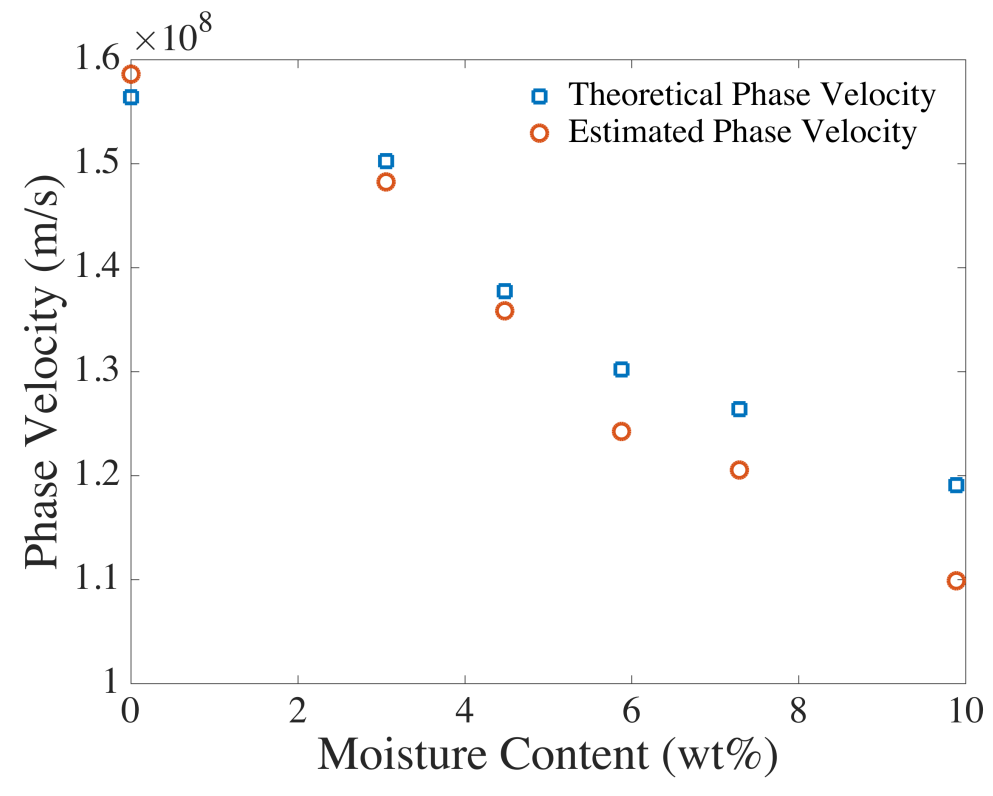

Figure 11: The theoretical and estimated phase velocities of the clay for different moisture levels using a1200 MHz Ricker wavelet source.

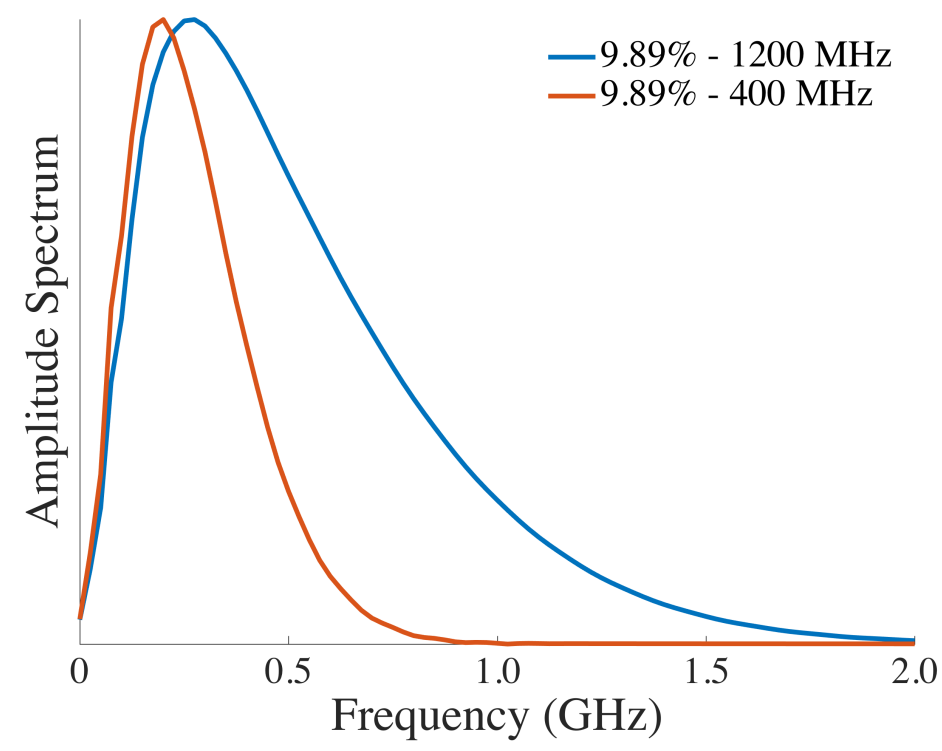

Figure 12: Amplitude spectra of the received signals from simulations using $1200 \mathrm{MHz}$ and $400 \mathrm{MHz}$ source. The centroid frequency is higher than spectral maximum for the frequencies since the spectra are not symmetric. 
We have shown that although the estimation from the spectral shift method is not very accurate, the idea of distinguishing two dispersive materials by measuring the difference between $\mathrm{Q}^{*}$ at two frequencies is verified numerically. There are several potential limitations to this technique. In practical applications, the subsurface environment is much more complex than a single homogeneous material. Moreover, heterogeneities within the material may give rise to scattering loss that does not correspond to the material properties. Most importantly, the materials need to have detectable change in $\mathrm{Q}^{*}$ at the chosen frequencies.

\section{Conclusion}

Through measurements of the STX-1b Ca-rich montmorillonite sample under various moisture levels, we have identified dispersive behaviours caused by water that are consistent with previous studies. The relaxation times were obtained by inverting the permittivity data using the Cole-Cole model. We also measured the complex permittivity of a pulp sample, mainly composed of pyrite and quartz, from a massive sulphide mine. The pyrite-quartz sample has dispersive behaviours that are independent of water, which can be distinguished from that of moist clays by estimating $\mathrm{Q}^{*}$ at two different frequencies. A suite of simulations with the inverted parameters was implemented using gprMax to verify this idea. Our results show that the spectral shift method correctly identifies the pattern of change in $\mathrm{Q}^{*}$ of these two samples at $400 \mathrm{MHz}$ and $1200 \mathrm{MHz}$. Instead of a target-locating technique, we demonstrate how radar signals can also be used to distinguish two actual dispersive behaviours. Application of the concept to field data will be conducted in the future.

\section{References}

[1] J. L. Davis and A. P. Annan, "Ground-penetrating radar for high-resolution mapping of soil and rock stratigraphy," Geophysical Prospecting, vol. 37, no. 5, pp. 531-551, July 1989, doi: 10.1111/j.1365-2478.1989.tb02221.x. 
[2] A. P. Annan, "Transmission dispersion and GPR," Journal of Environmental and Engineering Geophysics, vol. 1, no. B, pp. 125-136, January 1996, doi: 10.4133/jeeg1.b.125.

[3] G. Turner and A. F. Siggins, "ConstantQattenuation of subsurface radar pulses,” Geophysics, vol. 59, no. 8, pp. 1192-1200, August 1994, doi: 10.1190/1.1443677.

[4] K. Aki and B. Chouet, "Origin of coda waves: Source, attenuation, and scattering effects," Journal of Geophysical Research, vol. 80, no. 23, pp. 3322-3342, August 1975, doi: 10.1029/jb080i023p03322.

[5] J. H. Bradford, "Frequency-dependent attenuation analysis of ground-penetrating radar data," Geophysics, vol. 72, no. 3, pp. J7-J16, May 2007, doi: 10.1190/1.2710183.

[6] Y. Quan and J. M. Harris, "Seismic attenuation tomography using the frequency shift method," Geophysics, vol. 62, no. 3, pp. 895-905, May 1997, doi: 10.1190/1.1444197.

[7] R. Calvet, "Dielectric properties of montmorillonites saturated by bivalent cations," Clays and Clay Minerals, vol. 23, no. 4, pp. 257-265, September 1975, doi: 10.1346/ccmn.1975.0230401.

[8] G. Sposito and R. Prost, "Structure of water adsorbed on smectites," Chemical Reviews, vol. 82, no. 6, pp. 553-573, December 1982, doi: $10.1021 / \mathrm{cr00052a001.}$

[9] G. R. Olhoeft, "Electrical properties from 10-3 to 10+9 HZ-physics and chemistry," in AIP Conference Proceedings. AIP, March 1987, doi: $10.1063 / 1.36399$.

[10] T. Ishida, T. Makino, and C. Wang, "Dielectric-relaxation spectroscopy of kaolinite, montmorillonite, allophane, and imogolite under moist conditions," Clays and Clay Minerals, vol. 48, no. 1, pp. 75-84, January 2000, doi: 10.1346/ccmn.2000.0480110.

[11] A. Robert, "Dielectric permittivity of concrete between $50 \mathrm{mhz}$ and $1 \mathrm{ghz}$ and GPR measurements for building materials evaluation," 
Journal of Applied Geophysics, vol. 40, no. 1-3, pp. 89-94, October 1998, doi: 10.1016/s0926-9851(98)00009-3.

[12] G. Klysz, J.-P. Balayssac, and S. Laurens, "Spectral analysis of radar surface waves for non-destructive evaluation of cover concrete," NDT \& E International, vol. 37, no. 3, pp. 221-227, April 2004, doi: 10.1016/j.ndteint.2003.09.006.

[13] W. Lai, T. Kind, and H. Wiggenhauser, "Frequency-dependent dispersion of high-frequency ground penetrating radar wave in concrete," NDT \& E International, vol. 44, no. 3, pp. 267-273, May 2011, doi: 10.1016/j.ndteint.2010.12.004.

[14] J. H. Bradford, "Frequency dependent attenuation of GPR data as a tool for material property characterization: A review and new developments," in 2011 6th International Workshop on Advanced Ground Penetrating Radar (IWAGPR). IEEE, June 2011, doi: 10.1109/iwagpr.2011.5963870.

[15] P. J. W. Debye, Polar molecules. Chemical Catalog Company, Incorporated, 1929.

[16] K. S. Cole and R. H. Cole, "Dispersion and absorption in dielectrics i. alternating current characteristics," The Journal of Chemical Physics, vol. 9, no. 4, pp. 341-351, April 1941, doi: $10.1063 / 1.1750906$.

[17] S. Havriliak and S. Negami, "A complex plane representation of dielectric and mechanical relaxation processes in some polymers," Polymer, vol. 8, pp. 161-210, January 1967, doi: 10.1016/0032-3861(67)90021-3.

[18] M. Wollensack, J. Hoffmann, J. Ruefenacht, and M. Zeier, "VNA tools II: S-parameter uncertainty calculation," in 79th ARFTG Microwave Measurement Conference. IEEE, June 2012, doi: 10.1109/arftg79.2012.6291183.

[19] D. K. Rytting, "Network analyzer accuracy overview," in 58th ARFTG Conference Digest. IEEE, November 2001, doi: 10.1109/arftg.2001.327486. 
[20] A. M. Nicolson and G. F. Ross, "Measurement of the intrinsic properties of materials by time-domain techniques," IEEE Transactions on Instrumentation and Measurement, vol. 19, no. 4, pp. 377-382, November 1970, doi: 10.1109/tim.1970.4313932.

[21] W. Weir, "Automatic measurement of complex dielectric constant and permeability at microwave frequencies," Proceedings of the IEEE, vol. 62, no. 1, pp. 33-36, January 1974, doi: 10.1109/proc. 1974.9382 .

[22] A. H. Boughriet, C. Legrand, and A. Chapoton, "Noniterative stable transmission/reflection method for low-loss material complex permittivity determination," IEEE Transactions on Microwave Theory and Techniques, vol. 45, no. 1, pp. 52-57, February 1997, doi: $10.1109 / 22.552032$.

[23] P. L. Hall and D. M. Astill, "Adsorption of water by homoionic exchange forms of wyoming montmorillonite (SWy-1)," Clays and Clay Minerals, vol. 37, no. 4, pp. 355-363, August 1989, doi: 10.1346/ccmn. 1989.0370409.

[24] P. Bala, B. K. Samantaray, and S. K. Srivastava, "Dehydration transformation in ca-montmorillonite," Bulletin of Materials Science, vol. 23, no. 1, pp. 61-67, February 2000, doi: 10.1007/bf02708614. [Online]. Available: https://doi.org/10.1007/bf02708614

[25] A. Kuligiewicz and A. Derkowski, "Tightly bound water in smectites," American Mineralogist, vol. 102, no. 5, pp. 1073-1090, May 2017, doi: 10.2138/am-2017-5918.

[26] G. R. Olhoeft, "Low-frequency electrical properties," Geophysics, vol. 50, no. 12, pp. 2492-2503, December 1985, doi: $10.1190 / 1.1441880$.

[27] D. E. Stillman, "Frequency and temperature dependence in electromagnetic properties of martian analog materials," Ph.D. dissertation, Colorado School of Mines, Golden, Colo., 2006.

[28] D. Stillman and G. Olhoeft, "Frequency and temperature dependence in electromagnetic properties of martian analog minerals," Journal 
of Geophysical Research, vol. 113, no. E9, September 2008, doi: 10.1029/2007je002977.

[29] A. Benedetto, "Water content evaluation in unsaturated soil using GPR signal analysis in the frequency domain," Journal of Applied Geophysics, vol. 71, no. 1, pp. 26-35, May 2010, doi: 10.1016/j.jappgeo.2010.03.001.

[30] M. Newville, T. Stensitzki, D. B. Allen, and A. Ingargiola, "Lmfit: Non-linear least-square minimization and curve-fitting for python," 2014, doi: 10.5281/zenodo. 11813 .

[31] C. Warren, A. Giannopoulos, and I. Giannakis, "gprMax: Open source software to simulate electromagnetic wave propagation for ground penetrating radar," Computer Physics Communications, vol. 209, pp. 163-170, December 2016, doi: 10.1016/j.cpc.2016.08.020.

[32] K. Yee, "Numerical solution of initial boundary value problems involving maxwell's equations in isotropic media," IEEE Transactions on Antennas and Propagation, vol. 14, no. 3, pp. 302-307, May 1966, doi: 10.1109/tap.1966.1138693.

[33] I. Giannakis, A. Giannopoulos, and N. Davidson, "Incorporating dispersive electrical properties in FDTD GPR models using a general cole-cole dispersion function," in 2012 14th International Conference on Ground Penetrating Radar (GPR). IEEE, June 2012, doi: 10.1109/icgpr.2012.6254866.

[34] M. Loewer, J. Igel, and N. Wagner, "Spectral decomposition of soil electrical and dielectric losses and prediction of in situ GPR performance," IEEE Journal of Selected Topics in Applied Earth Observations and Remote Sensing, vol. 9, no. 1, pp. 212-220, January 2016, doi: 10.1109/jstars.2015.2424152.

[35] F. Uddin, "Clays, nanoclays, and montmorillonite minerals," Metallurgical and Materials Transactions A, vol. 39, no. 12, pp. 2804-2814, September 2008, doi: 10.1007/s11661-008-9603-5. 
The scientific paper that you have downloaded is included in Issue 1, Volume 2 (March 2019) of the journal Ground Penetrating Radar (ISSN 2533-3100; journal homepage: www.gpradar.eu/journal).

All Ground Penetrating Radar papers are processed and published in true open access, free to both Authors and Readers, thanks to the generous support of TU1208 GPR Association and to the voluntary efforts of the journal Editorial Board. The publication of Issue 1, Volume 2 is also supported by IDS Georadar s.r.1. (idsgeoradar.com). The present information sheet is obviously not part of the scientific paper.
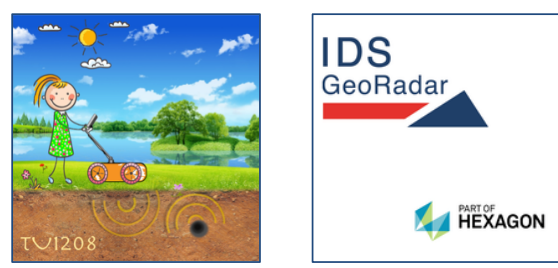